\title{
Slipped Capital Femoral Epiphysis: A Case Report and Comprehensive Review of Current Literature
}

\author{
Rachel Michael, MD, Christopher Jackson, MD, Briana Stirling, MD, Vithal Shendge, MD \\ The University of Toledo Medical Center, 3000 Arlington Ave Toledo, OH 43614. \\ Rachel.Michael@utoledo.edu;Christopher.Jackson4@utoledo.edu \\ Briana.Thompson@utoledo.edu;drvits@gmail.com
}

\begin{abstract}
We present a case report to portray the possible complications of neglected and insitu treated SCFE, and treatment to enable correction of the deformities using total hip arthroplasty. We also conduct a mini review of literature to revisit the epidemiology, pathophysiology, clinical evaluation, and radiographic analysis of slipped capital femoral epiphysis.

This article also highlights the current literature and recommended treatments for slipped capital femoral epiphysis and sequelae associated with this disorder.
\end{abstract}

\section{INTRODUCTION}

Slipped capital femoral epiphysis (SCFE) is a condition seen in the adolescent population. Due to its association with obesity, the incidence of SCFE is rising due to the concurrent rise in adolescent obesity in America. However, although obesity is the greatest risk factor, other risk factors for SCFE include endocrinopathies (e.g. hypothyroidism, hypoparathyroidism, vitamin D deficiency, and hormone therapy), femoral retroversion, and history of radiation to the femoral head.

Epidemiologically, males are affected more frequently than females (2:1) and African American adolescents are more commonly affected than those of Caucasian descent $(2: 1)^{1,2}$. Adolescents of Polynesian and Latin American descent also have an increased incidence compared to those of Caucasian ancestry ${ }^{1,2}$.

Slipped capital femoral epiphysis occurs in a select age groups (12-15 years old in males and 11-13 years old in females) due to the physiology of the proximal femoral physis in relation the adolescent growth spurt ${ }^{1,2,3,4}$. Due to the relation of peak height velocity and growth remaining, SCFE rarely occurs in postmenarcheal females. If SCFE occurs outside of the expected age range, in an underweight patient, or outside of peak height velocity, it is important to assess for other metabolic causes such as endocrinopathies.

Often, SCFE can occur bilaterally. Bilateral slips are noted on initial presentation about $50 \%$ of the time ${ }^{1,4}$. If only one hip is affected on initial presentation there is a $17-50 \%$ chance of the contralateral hip becoming affected in the first 18 months following in the initial slip. Due to this incidence, close clinical and radiographic follow up is necessary. Additionally, it is sometimes recommended that the contralateral hip be treated prophylactically at the time of initial slip if patient has significant risk factors. Risk factors for contralateral SCFE include open triradiate cartilage and endocrinopathies.

\section{CASE REPORT}

\section{History}

A 23-year-old male presented with history of left chronic slipped capital femoral epiphysis that was treated at 
age of 14yrs with in situ screw fixation. The patient states that he has had pain since the occurrence of the SCFE, and has been progressive over time. His pain currently occurs at rest and is worse with activity and ambulation. His pain is sharp and severe and is located over the anterior groin. He also notes limitation in left hip range of motion, especially with extension and internal rotation. Patient has now failed conservative treatment with physical therapy, ambulatory assistive device, activity modification, and use of non-steroidal anti-inflammatory medication as well as narcotics. The patient currently denies any contralateral hip problems. He also denies any numbness, tingling, or weakness. The patient's medical history is otherwise negative with the exception of his SCFE history. His social history is pertinent for current daily tobacco use.

\section{Physical Exam}

On examination the patient is a 23-year-old male who presents with a grotesque left lower extremity deformity along with profound derangement in his gait as well as seated posture. Focused exam of the left lower extremities demonstrated intact skin circumferentially with fixed flexion deformity of 10 degrees, fixed abduction of 10 degrees and external rotation deformity of about 40 degrees approximately. Patient was found to have tenderness over the left greater trochanteric bursa and anterior groin pain with attempted internal rotation. Hip range of motion was then measured and is documented in Table 1. The patient was also noted to have a positive Drehmann sign of the left hip which demonstrated resultant external rotation of the hip with passive flexion.

Table1. Preoperative bilateral hip range of motion.

\begin{tabular}{|l|c|c|}
\hline \multicolumn{2}{|c|}{ Preoperative Hip Range of Motion } \\
\hline Hip Extension & Left & Right \\
\hline Hip Flexion & $5^{\circ}$ & $-5^{\circ}$ \\
\hline Hip Internal Rotation & $80^{\circ}$ & $110^{\circ}$ \\
\hline Hip External Rotation & $0^{\circ}$ at $80^{\circ}$ flexion & 25 \\
\hline Hip Abduction & $70^{\circ}$ (fixed) & 45 \\
\hline Hip Adduction & 15 & 40 \\
\hline
\end{tabular}

The patient was found to have full tone and motor strength in flexion and adduction of the bilateral lower extremities. However, it was noted that his left hip abduction was weak compared to the contralateral side, with normal sensations in bilateral lower extremities.

The patient's stance and gait were also observed and demonstrated varus alignment of the left knee with calcaneo-planovalgus deformity of the left ankle and foot with loss of the plantar medial arch. Patient walks with an antalgic Trendelenburg gait with evident left gluteal muscle wasting and contracture of left hip abductors and external rotators following his flexion, abduction and external rotation deformity as a sequelae of his left hip SCFE. He also walks with an external foot progression bilaterally but with enhanced external foot progression on the left lower extremity as demonstrated in Figure 1.

Radiographs of the patient's pelvis, left hip, and full-length lower extremities were obtained. Figure 2 illustrates the radiographic images that demonstrate left hip in situ screw fixation with collapse of the left femoral head and coxa magna, along with secondary degenerative changes in the femoral head and acetabulum with an external rotation alignment are also seen. Right hip shows degenerative changes to a lesser extent.

American Research Journal of Orthopedics and Traumatology 
Slipped Capital Femoral Epiphysis: A Case Report and Comprehensive Review of Current Literature

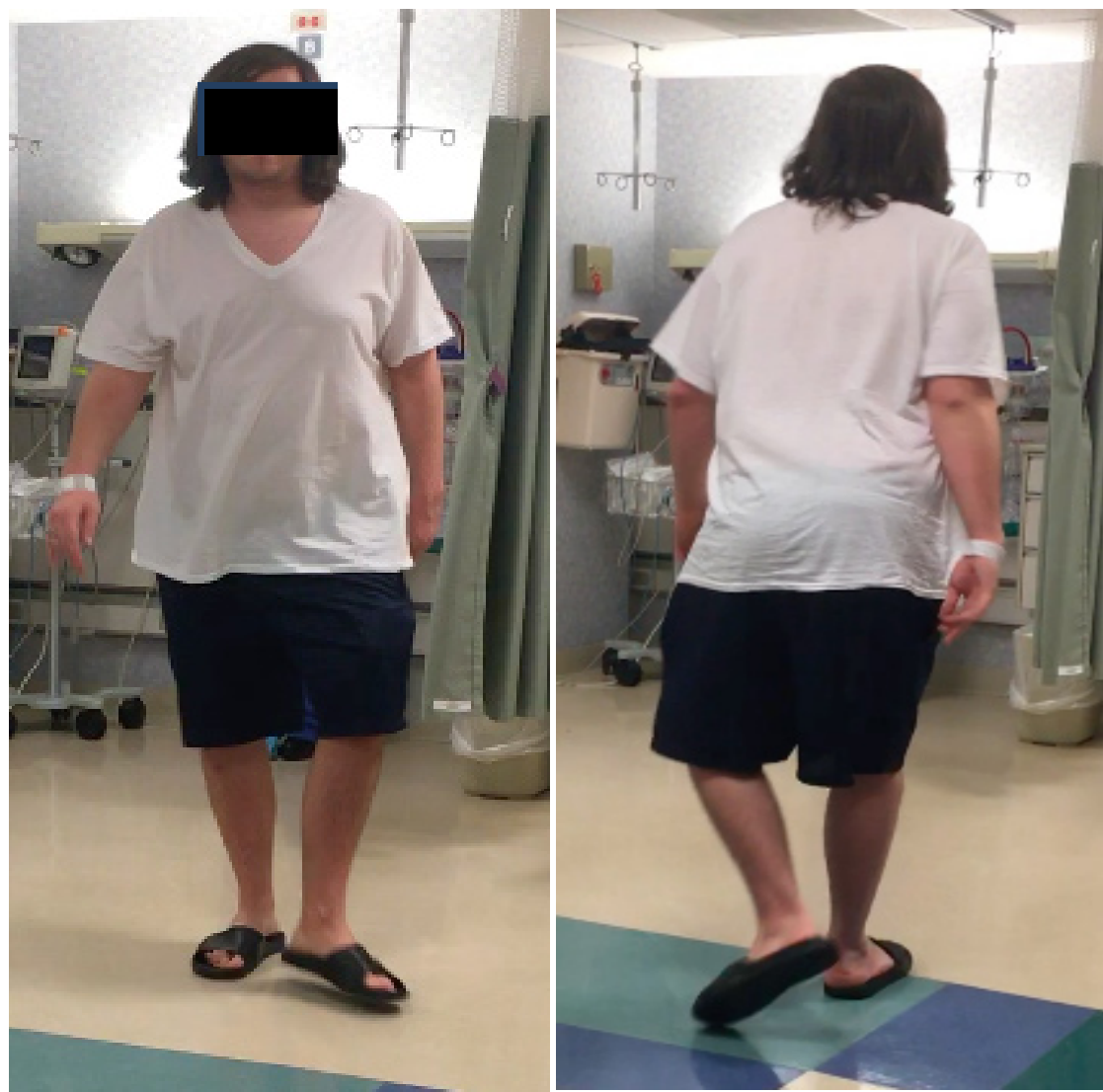

Figure1. Gait photos demonstrating external foot progression bilaterally but with enhanced external foot progression on the left lower extremity as well as left hip flexion contracture.

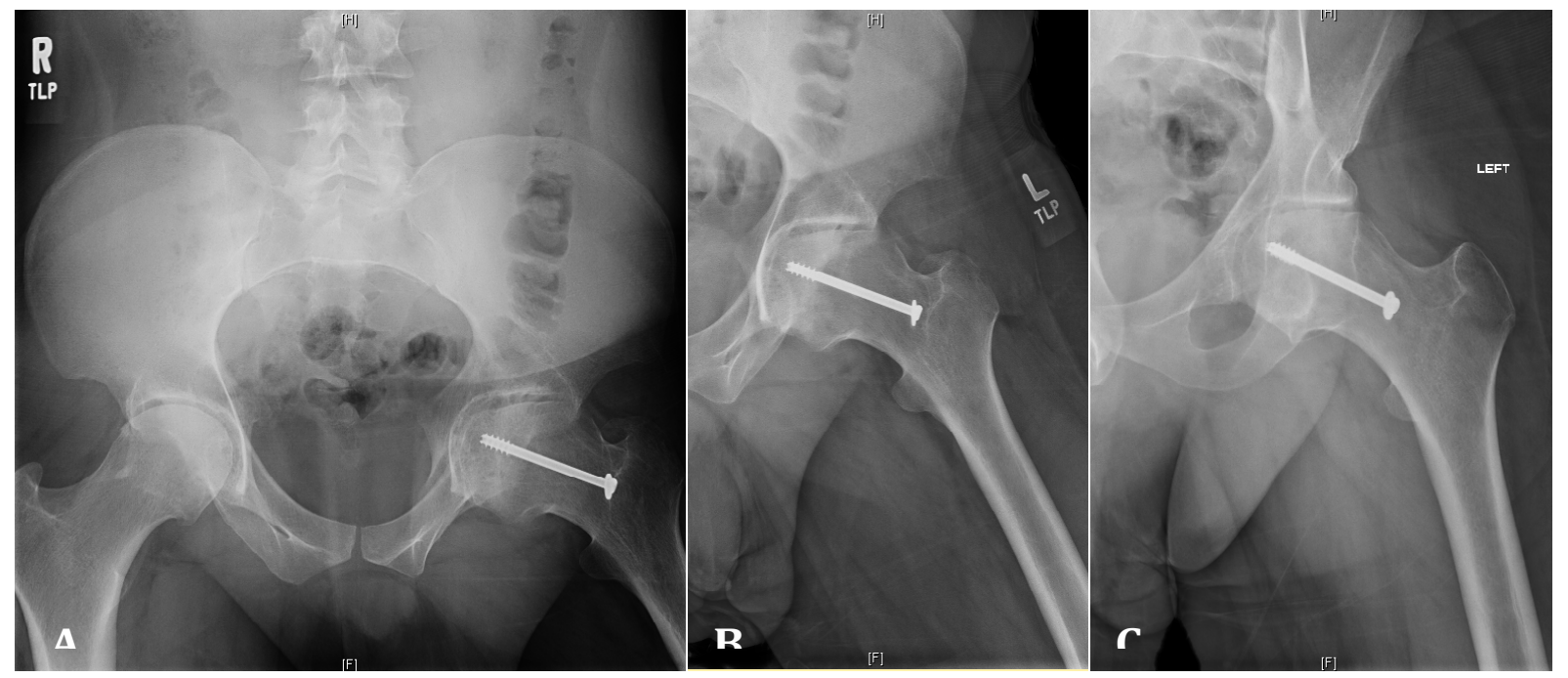

Figure 2. Demonstrates our patients presenting radiographs nine years after left hip in situ screw fixation. Image $A$ represents the AP pelvis view. Images B-C are the AP and lateral views of the left hip respectively. The radiographs demonstrate collapse of the left femoral head with Coxa magna and significant degenerative changes in the femoral head and acetabulum with an external rotation alignment as depicted by the lesser trochanteric visualization, and hypoplasia of the left femoral shaft Right hip has minimal degenerative changes. 


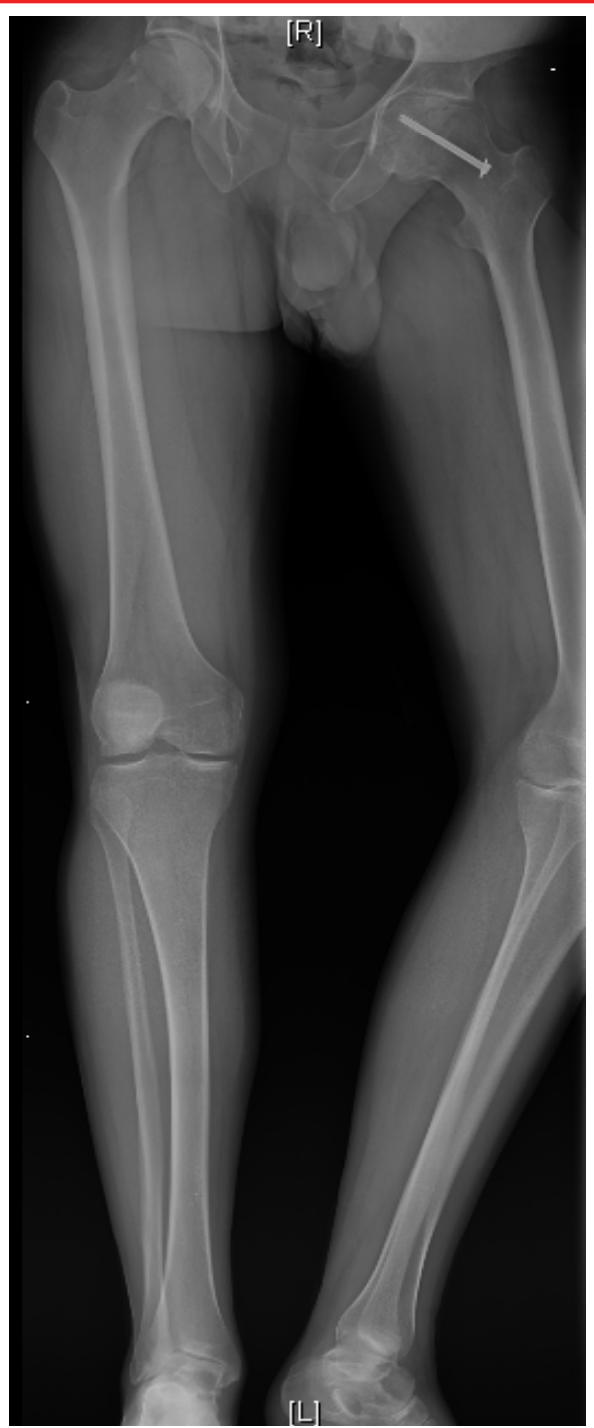

Figure3. Represents a full-length lower extremity joint survey radiograph. This joint survey shows flexed posturing of left knee and hip joints withvalgus external rotation of the hip and compensatory varus external rotation deformity of the knee joint along with equinus of the ankle. Limb length discrepancy of $1 \mathrm{~cm}$ was measured on the left lower extremity due to flexion deformity however, weight bearing axis limb length difference measured almost $4 \mathrm{cms}$.

Joint survey of the bilateral lower extremities as seen in Figure 3 demonstrates flexed posturing of left knee and hip joints with valgus external rotation of the hip and compensatory varus external rotation deformity of the knee joint along with equinus of the ankle. Limb length discrepancy of $1 \mathrm{~cm}$ was measured on the left lower extremity due to flexion deformity however; weight bearing axis limb length difference measured almost 4 cms.

Due to the complex nature of the patient's deformity, a CT scan of the pelvis and lower extremities was obtained for further assessment and treatment planning. Axial cuts of the CT scan are illustrated in Figure 4. The CT scan showed 18 degrees of external rotation on the right femur and 38 degrees of external rotation on the left femur. Femoral retroversion was also noted on the left hip with coxa valgus of 47 degrees on the left and 50 degrees on the right. Femoral length was measured as $51 \mathrm{~cm}$ bilaterally. 


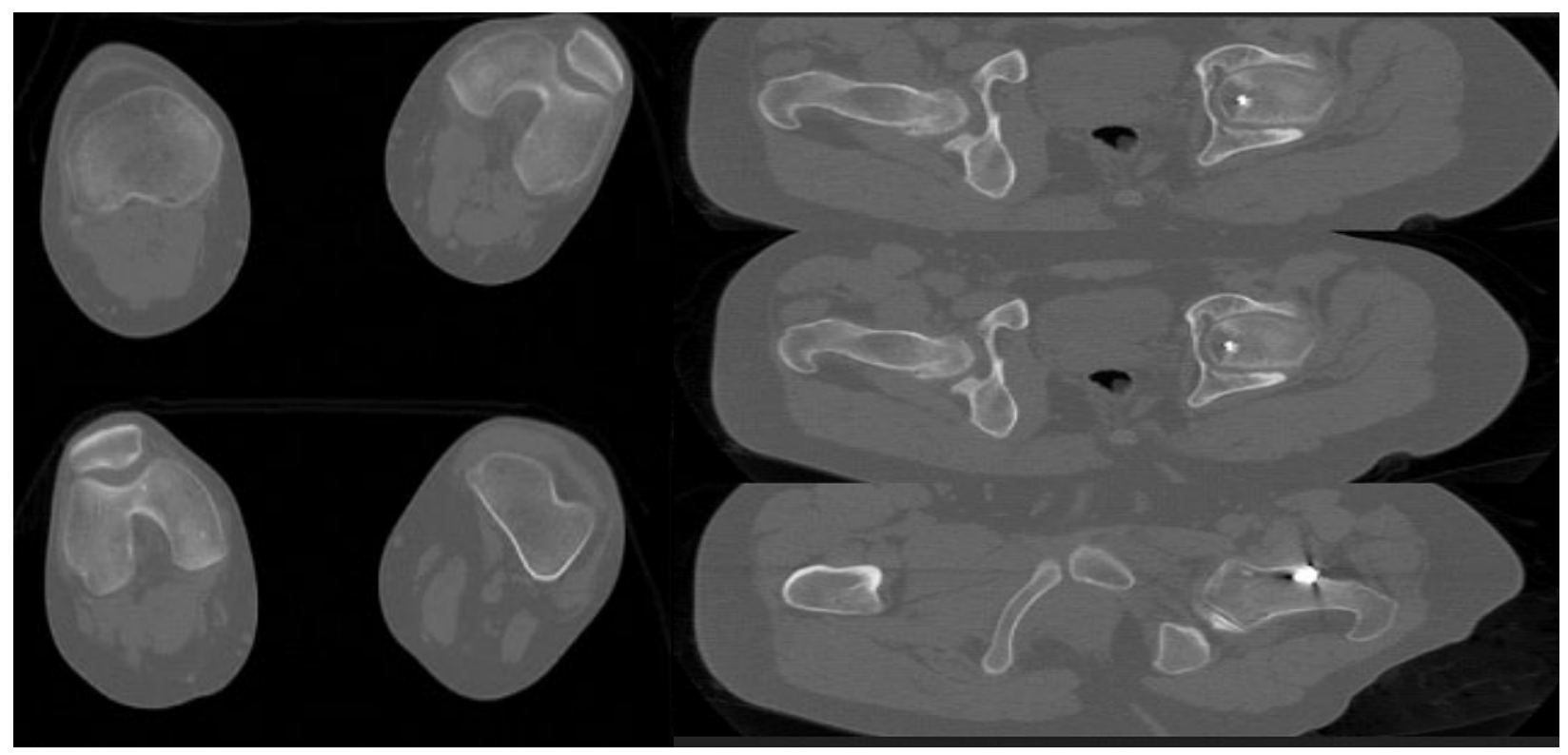

Figure4. Axial CT images of bilateral lower extremities. The right side measured 18 degrees of external rotation; left side is 38 degrees of external rotation. Femoral retroversion was noted on the left with coxa valgus of 47 degrees on the left and 50 degrees on the right. Femoral length is $51 \mathrm{~cm}$ bilaterally.

Plan

Since the patient had failed conservative measures at this time, total hip arthroplasty was recommended. Due to his poor function and quality of life due to pain and deformity of his left hip, and after being explained the risks, benefits, and alternatives, the patient elected to proceed with hardware removal with subsequent left total hip arthroplasty.

Prior to surgical intervention, patient was counseled on smoking cessation, and underwent physical therapy for targeted gluteal muscle strengthening to decrease postoperative complications. Patient successfully quit smoking prior to surgery. We also discussed with the patient the possibility of femoral derotation osteotomy to aid in deformity correction. However, due to the patients main complaint of hip pain rather than gait disturbance and due to the patient's remote smoking history it was agreed upon to proceed with total hip arthroplasty.

\section{Surgical Course}

Prior to sterile draping, the patient's fixed deformity was evaluated and documented in the lateral position (Figure 5). The patient was immobilized in lateral position with the use of a peg board and a posterior lateral approach to the hip was utilized. Care was taken to protect sciatic nerve as his femoral neck was extremely retroverted thus putting our approach trajectory to the hip more posterior than normal. Intraoperatively, the abductors were found to be extremely contracted with hypertrophy of the pulvinar and capsule on the anterior aspect however we were able to tag and later repair piriformis to its anatomic insertion.

Once we incised through the capsule, we performed our surgical hip dislocation and then made our femoral neck osteotomy at $10 \mathrm{mms}$ above the lesser trochanter. The femoral head was subsequently removed without difficulty as our neck cut was made below the level of his retained hardware (Figure 6). We then prepared the acetabulum and placed the cup. We placed a single acetabular screw in the posterior superior quadrant. We then placed the liner. We then prepared the femur and placed a size 5 femoral stem with a neck length of $35 \mathrm{~mm}$ and stem length of 108 . A dual modular ceramic size $28 \mathrm{~mm}$ head with $0 \mathrm{~mm}$ neck length was then implanted. 
Slipped Capital Femoral Epiphysis: A Case Report and Comprehensive Review of Current Literature

Dual modular components were utilized due to the patient's high risk of dislocation related to severe wasting of his left abductor muscles (Figure 7)

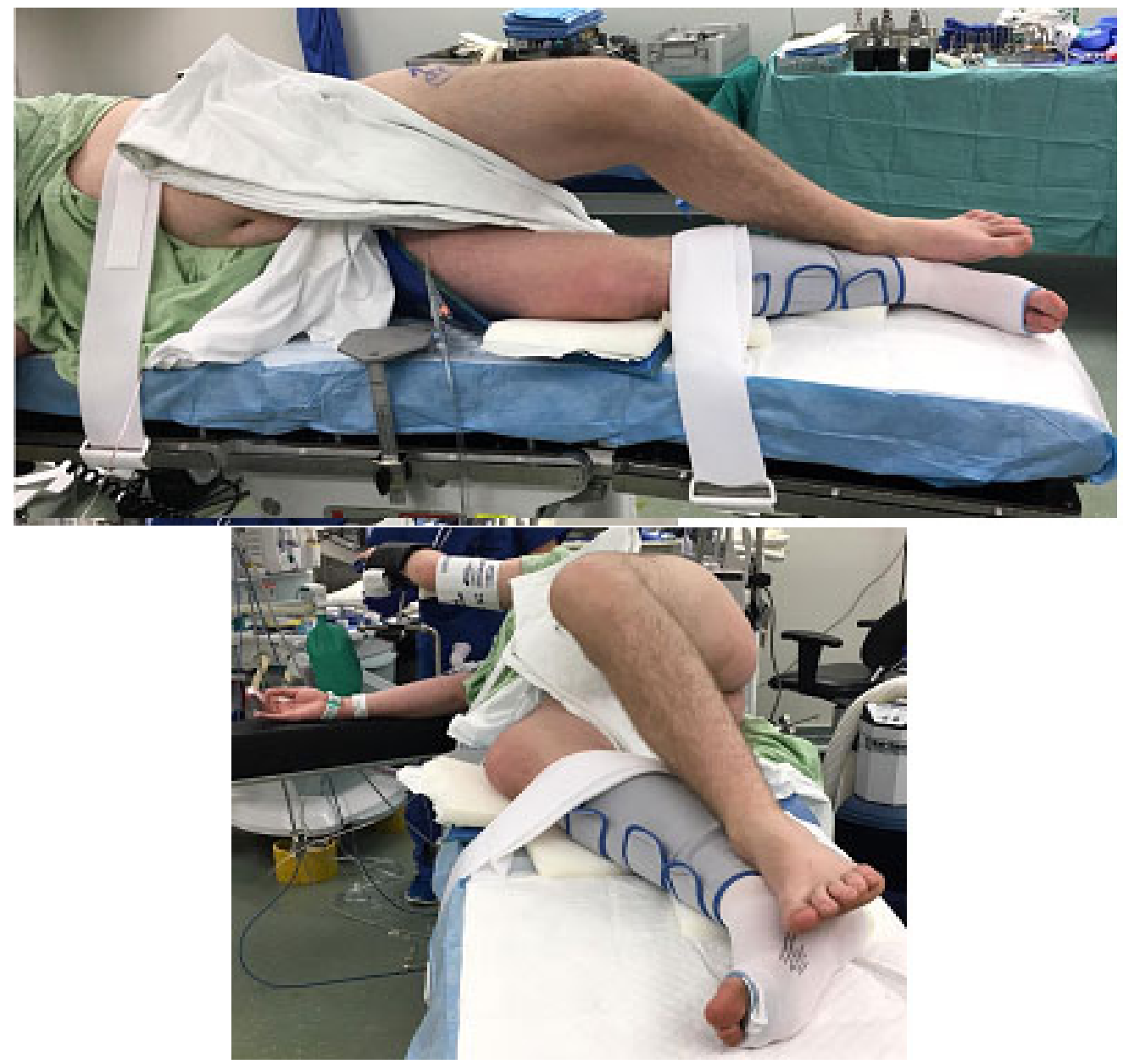

Figure5. Intraoperative images of lateral positioning of the patient. These images demonstrate the severity of the fixed nature of the left hip flexion, abduction, and external rotation deformity (FABER) a sequela of non-reduced SCFE

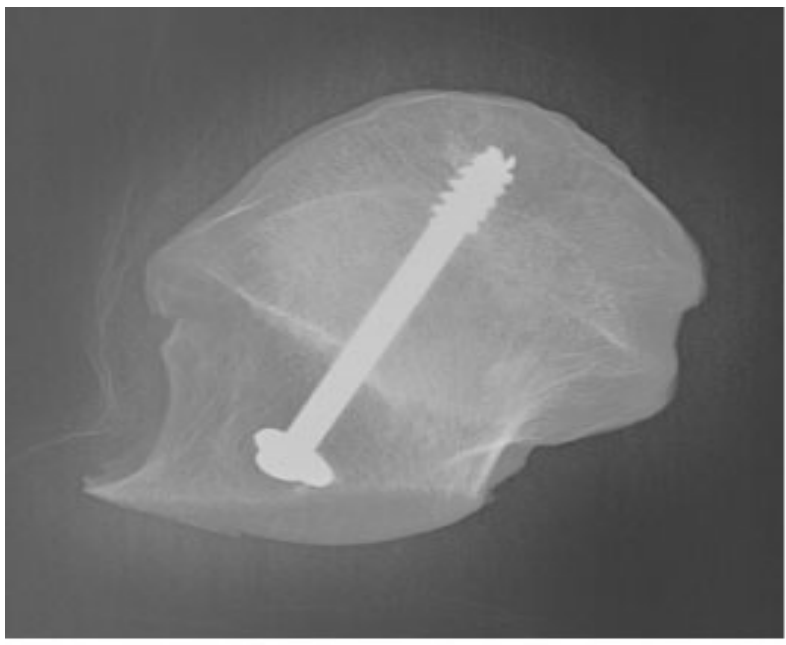

Figure6. Radiograph of the removed left femoral head containing the patient's prior in situ screw. 


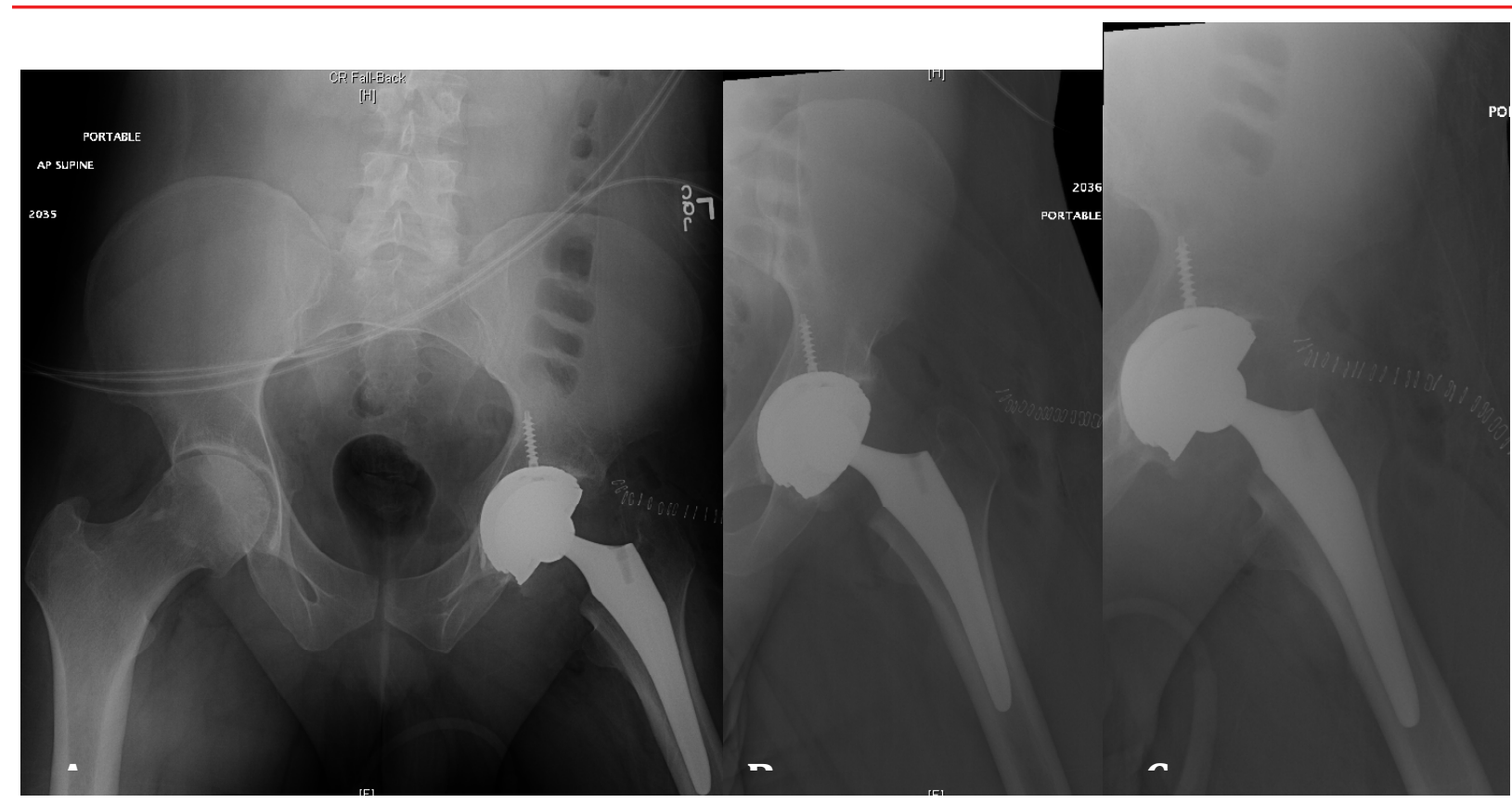

Figure7. Demonstrates the postoperative radiographs of the AP pelvis (image A), and AP and lateral of the left hip (images $B$ and $C$ respectively). Dual mobility components were implanted.

\section{Follow Up}

At 2 week postoperative follow-up visit, patient had commenced physical therapy and gait training and is very happy with his deformity correction being able to ambulate like normal person after more than a decade. At this time, patient was using a cane in his opposite hand for ambulation (Figure 8). He denies any pain in the left groin. He denies any noticeable or limiting limb length discrepancy. Overall, he is pleased with the surgery. Physical examination at 2 weeks postoperatively demonstrated a well-healed incision and patient ambulatory without antalgia. Postoperative hip range of motion was assessed and is illustrated in Table 2. Also, he continues to have very mild external rotational deformity and with a foot progression angle of about $15^{\circ}$. Radiographs of the pelvis and left hip were also obtained 2 weeks postoperatively and demonstrated well-maintained component alignment (Figure 9). At this point, it was recommended for the patient to continue gait training with physical therapy for an additional 4-6 weeks. He will also continue with posterior hip and fall precautions.

Table2. Postoperative bilateral hip range of motion

\begin{tabular}{|l|c|c|}
\hline \multicolumn{2}{|c|}{ Postoperative Hip Range of Motion } \\
\hline & Left & Right \\
\hline Hip Extension & $10^{\circ}$ & $-5^{\circ}$ \\
\hline Hip Flexion & $90^{\circ}$ & $110^{\circ}$ \\
\hline Hip Internal Rotation & $15^{\circ}$ & 10 \\
\hline Hip External Rotation & $30^{\circ}$ & 35 \\
\hline Hip Abduction & $40^{\circ}$ & 35 \\
\hline Hip Adduction & $25^{\circ}$ & 25 \\
\hline
\end{tabular}

American Research Journal of Orthopedics and Traumatology 
Slipped Capital Femoral Epiphysis: A Case Report and Comprehensive Review of Current Literature

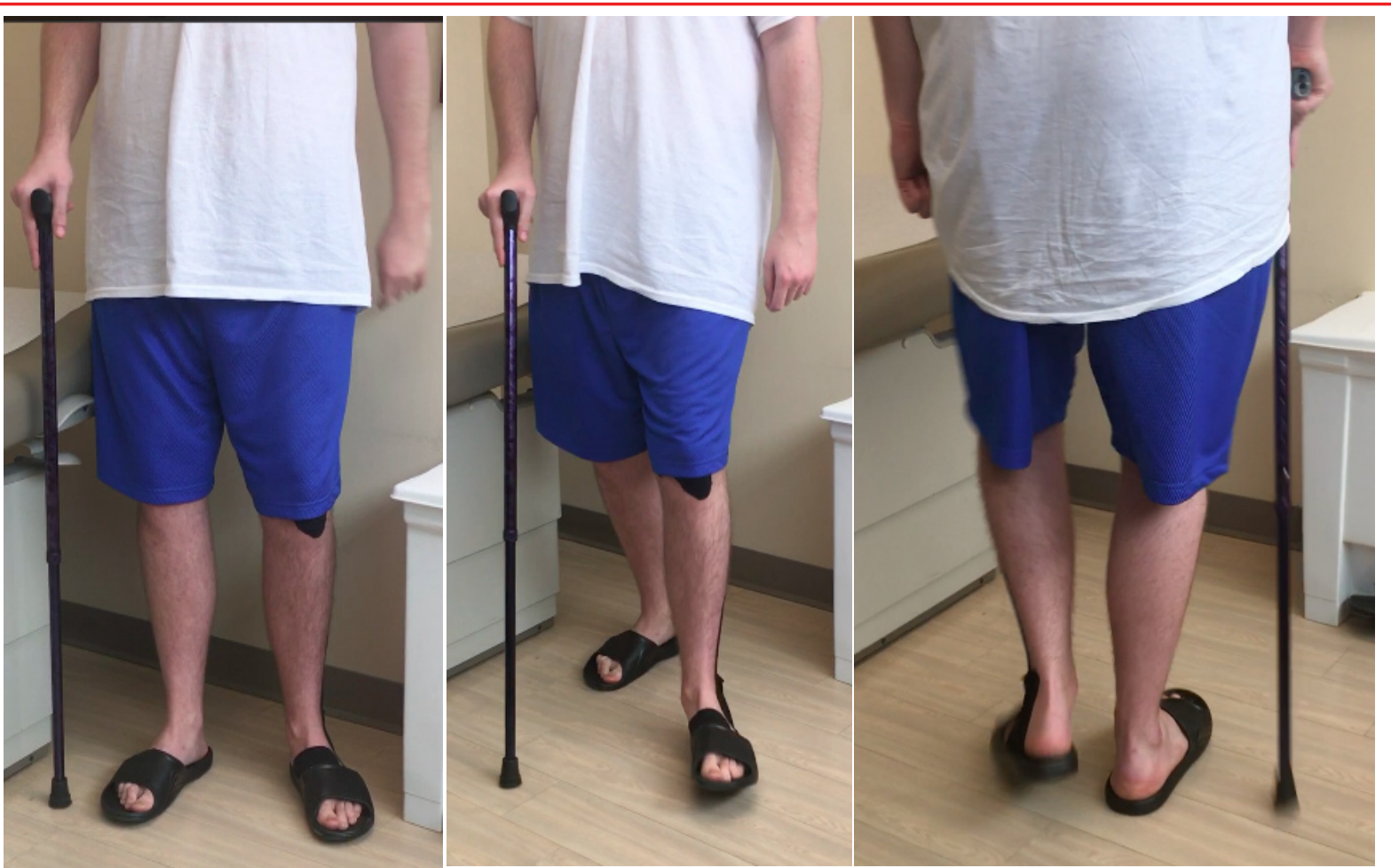

Figure8. At 2 weeks post operatively, patient was ambulating with cane assistance.

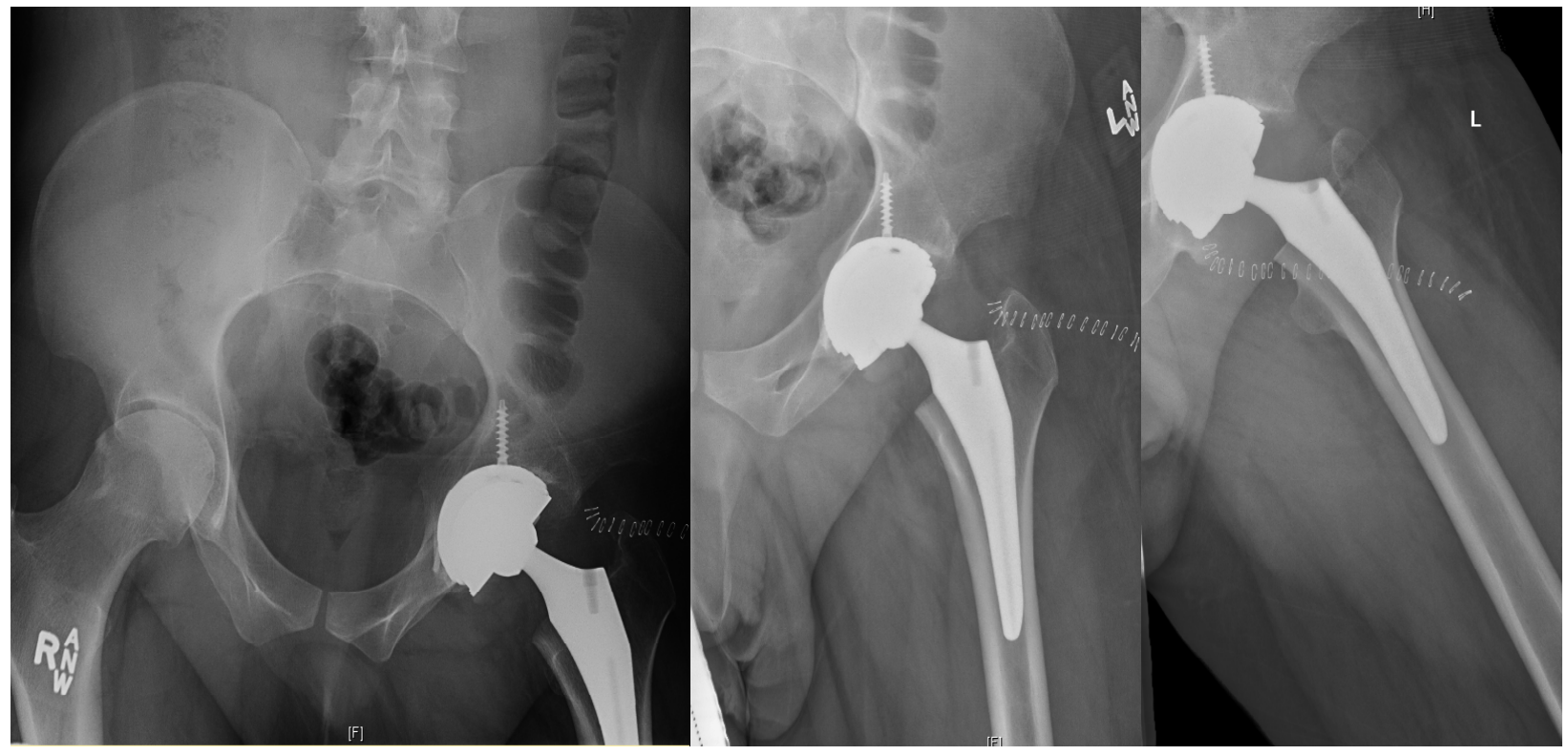

Figure9. Demonstrates the 2 week postoperative radiographs of the AP pelvis and AP and lateral of the left hip. Components remain well-aligned at this time.

\section{Postoperative Follow Up}

The patient was seen at two weeks, 6 weeks, 2 months and 6 months post-operatively and continued to have progressive improvement in his gait pattern, range of motion of the hip joint as well as strength. He is now walking without any assistive devices and is overall pleased with his normalized gait pattern (Figure 10). 


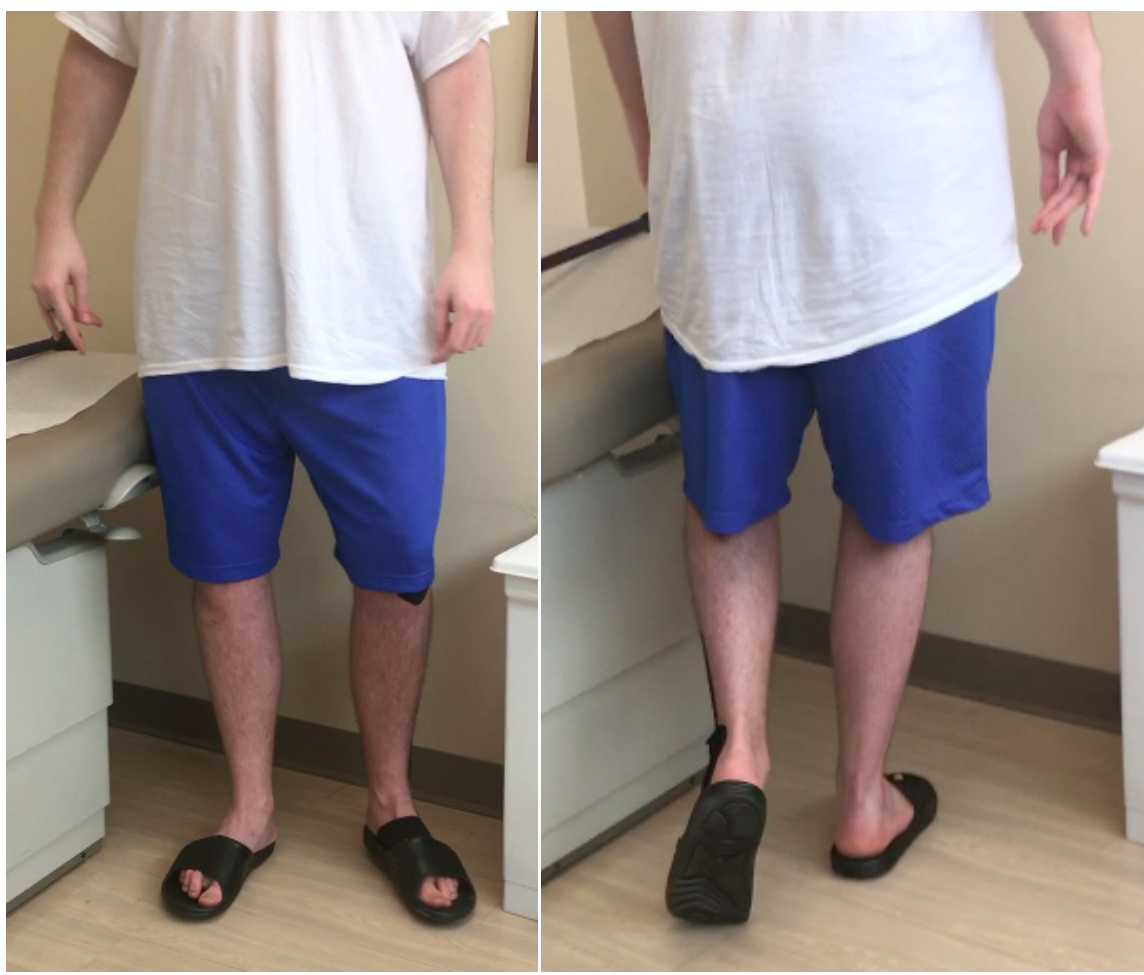

Figure10. Normalization of gait pattern without assistive device at 6 weeks postoperatively.

\section{Pathophysiology}

\section{The Physis ${ }^{5,6}$}

Normal physeal anatomy can be subdivided into four different layers or zones, starting from the epiphysis and extending to the metaphysis. Growth plates produce longitudinal growth of the bone. Zone 1 is the "reserve zone" located adjacent to the epiphysis and contains inactive cells and allows for matrix production. Zone 2 is the "proliferating zone" and is used for cellular proliferation and longitudinal growth. Zone 3 is the "hypertrophic zone" and contains chondrocytes that are large and more organized. This zone is subdivided into the zones of maturation, degeneration and provisional calcification. The majority of growth plate injuries occur in the hypertrophic zone. This zone is the weakest, providing a cleavage pathway for fractures or physeal slips. Zone 4 is the final layer "the zone of calcification" where cartilage is calcified and begins to remodel into bone.

\section{Physeal Pathology in SCFE}

During rapid growth in adolescence, there is weakening of the proximal femoral physis with resultant shearing stress that causes a physeal slip through the hypertrophic zone ${ }^{1,7}$. The shearing stresses at this time of high growth velocity are accentuated in overweight individuals. Anatomically there is a fibrous band that encases and supports the physis at the cartilage-bone junction called the perichondral ring. Physeal slips are also a result of thinning of this perichondral ring in adolescence, leading to loss of structural support ${ }^{1}$.

When slipped capital femoral epiphysis occurs, it results in displacement of the femoral neck relative to the femoral capital epiphysis. The most common direction of dislocation is with the epiphysis posterior to the neck ${ }^{1,7}$. The metaphysis also undergoes external rotation at the time of dislocation.

In examining the physeal histology in SCFE, the "pre-slip" stage demonstrates edema within the synovial membrane, capsule, and periosteum with thickening of the proliferative and hypertrophic zones ${ }^{1,7,8}$. Resting 
zone at this stage has normal histology. After the occurrence of a slipped capital femoral epiphysis, histology demonstrates decreased quantity of chondrocytes with disruption of their columnar alignment in addition to excessive matrix in proliferative and hypertrophic zones ${ }^{1,7}$.

\section{Clinical Evaluation/Classification}

Clinical evaluation should always begin with a thorough history and physical examination. The history of the patient's symptoms in slipped capital femoral epiphysis help to classify the injury and aid in treatment and prognosis. The Loder classification of SCFE include stable versus unstable slips ${ }^{1,2,7}$. A stable slip is demonstrated by the ability to bear weight on the affected extremity with or without crutches. A stable slip is associated with $<10 \%$ risk of avascular necrosis ${ }^{1,2}$. In comparison, an unstable slip is classified as the inability to weight bear even with the aid of crutches. An unstable slip is associated with a $47 \%$ risk of avascular necrosis ${ }^{1,2}$.

The patient's history of symptom duration also classifies the slip as acute versus chronic. Symptoms duration for less than 3 weeks is considered acute, and a symptom greater than 3 weeks demonstrates a chronic slip ${ }^{1,2,7}$.

On physical examination, the patient gait or inability to weight bear should be observed. If able to ambulate, an external foot progression may be observed indicating femoral retroversion. Range of motion of the affected hip may demonstrate restricted internal rotation, abduction, and flexion. Patients that note pain at the limit of internal rotation have a more unstable slip. The Drehmann sign is an examination sign specific for SCFE. A positive Drehmann sign is when the hip externally rotates with passive hip flexion ${ }^{1}$. This occurs due to impingement of the femoral metaphysis on the acetabulum. The contralateral hip should also be assess in every patient as well as a thorough neurovascular examination.

\section{Radiographic Analysis}

All patients with concern for slipped capital femoral epiphysis should have an AP and frog lateral radiograph following physical examination. The frog lateral view is the most sensitive in detecting early slips. Radiographically, chronic slips will demonstrate some degree of femoral neck remodeling which is not evident in cases of an acute slip.

Radiographic measures are also used for classifying the severity of the slip. Slips can be graded by percentage of physeal involvement, angle of displacement, and metaphyseal overlap. In the Wilson grading system, a grade 0 slip involves epiphysiolysis which leads to physeal widening with no evidence of displacement ${ }^{9}$. Grade I slip is displacement of $0-33 \%$ of the physis. Grade II slip is displacement of $34-50 \%$ of the physis ${ }^{9}$. Grade III slip is when $>50 \%$ of the physis is displaced ${ }^{9}$. The angle of displacement is measured via the Southwick angle. The Southwick angle is calculated by the head to shaft angle difference from unaffected side, typically measured on the frog lateral view ${ }^{1,10}$. A mild slip is $<30$ degree difference, moderate $30-50$ degree difference, and severe $>50 \%$ difference from normal side ${ }^{1,10}$. If bilateral hips are involved upon initial presentation, measured angles are compared to averaged normal values. The normal head shaft angle on the frog lateral view should be 10 degrees. Another radiographic measure is the Klein line. This is a tangential line to the superior femoral neck. Normally, this line should bisect a small portion of the capital epiphysis. In cases of SCFE the line will not intersect the capital epiphysis due to the posterior dislocation of epiphysis ${ }^{1,9}$. Additionally, the metaphyseal blanch sign also known as the Steel sign can be observed. This is a crescent-shaped area of increased density that overlays the metaphysis next to the physis on AP radiograph ${ }^{1,9}$. The observed density is due to the overlap of the capital epiphysis and femoral neck.

In addition to plain radiography, additional imaging studies can be performed to assist a physician with the management of a SCFE. While plain radiography, history and physical examination are effective in diagnosing a SCFE, more advanced imaging can be used for surgical planning and to identify post-operative complications.

American Research Journal of Orthopedics and Traumatology

Page 10 
First ultrasonography can be used as an alternative to diagnosis through identification of joint effusions and an epiphyseal/metaphyseal step off. However, this modality has been shown to be no more effective than a true frog-leg lateral radiographs in diagnosis of SCFE ${ }^{11}$.

Computer tomography (CT) can also be utilized in the management of SCFE. As with ultrasonography, CT scans play a very minor role in diagnosis, but can assist with surgical management and the monitoring of postoperative complications. It has been shown that CT scans in an axial/sagittal oblique plane; parallel to the femoral neck is the most accurate image for determining posterior displacement and femoral retroversion ${ }^{12}$. Another benefit of CT is to determine the presence joint screw penetration postoperatively due to limitations of plain radiographs secondary to limited hip range of motion or obesity ${ }^{1}$.

Magnetic resonance imaging (MRI) and technetium-99 bone scans can similarly be utilized to monitor for post-operative complications. While bone scan is usually not necessary in diagnosis, increased uptake in the proximal femur will be the predominant feature in the setting of a SCFE. Bone scans can also reveal decreased uptake in the femoral head with the development of avascular necrosis (AVN) and increased uptake in both the femur and acetabulum in the presence of chondrolysis ${ }^{1}$. In addition, pretreatment bone scans have been shown to be predictive of 12 -month AVN in unstable SCFEs ${ }^{13}$. In terms of MRI, a diagnosis of a "pre-slip" SCFE can be made, which is characterized by physeal widening on T1 imaging with associated synovitis and bone marrow edema on T2 imaging ${ }^{14}$. Secondly, MRI can be useful in the diagnosis of postoperative AVN, however the presence of orthopedic hardware may significantly impair the accuracy of this modality and may require implant removal prior to MRI.

\section{Treatment}

Initial treatment of a suspected SCFE should include immobilization with wheelchair or stretcher ambulation followed by immediate radiographic imaging. Once confirmation of the diagnosis is made, the patient should be admitted to the hospital with bed rest restrictions until definitive fixation can be performed. This level of caution is necessary due to the possibility of developing an acute-on-chronic SCFE which can create further displacement and thus increase the patient's risk of AVN and poorer outcomes ${ }^{15,16}$. Overall treatment options are dictated by whether the SCFE is considered stable or unstable.

Overall, the gold standard for treatment of stable SCFE is in situ screw fixation while there are multiple described techniques, this paper will focus on several key aspects that should be followed. Typically large diameter (6.5 or $7.3 \mathrm{~mm}$ ) cannulated partially threaded screws are used. Next, the starting point for percutaneous fixation should be considered. Using fluoroscopic imaging, the typical starting point in a SCFE is found to more anterior and superior than a traditional laterally placed femoral neck screw ${ }^{17}$. Failure to achieve a proper starting point can result in posterior femoral neck perforation and inadequate epiphyseal fixation. Next, the surgeon should consider the orientation and configuration of the screw placement. In terms of screw configuration, a single screw configuration yields good results $18,19,20,21$. While multiple pin/screw constructs have been found to have increased stiffness and stability, this must be weighed with the increased risk of screw complications ${ }^{1}$. Stambough et.al. reported a five fold increase in complications with the addition of a second screw ${ }^{22}$. However, other authors reported only 20-33\% increased stiffness with a 2-screw construct ${ }^{23}$. Positioning of the screw should be aimed to be center-center on orthogonal views intraoperatively. However, slight inferoposterior positioning has been shown to decrease screw related complication. Care should be taken to avoid a superior/ anterior screw placement as this will also increase intra-articular screw penetration or progression of deformity due to inadequate fixation ${ }^{23}$. Finally, the screw should be advanced to achieve at least 3 threads of fixation within the femoral epiphysis. One study examining slip progression following single screw fixation of SCFE found that all case of slip progression occurred with fewer than 5 threads of epiphyseal fixation on postoperative frog-leg lateral radiographs and no cases of progression in patient with $\geq 5$ threads of fixation ${ }^{24}$.

American Research Journal of Orthopedics and Traumatology

Page 11 
Post-operatively, the patient should be placed on restricted weight bearing for greater than 6 weeks with routine radiographs to monitor for slip progression. One area that is controversial in SCFE treatment concerns the removal of orthopedic implants. Typically, hardware should remain in place until physeal closure to limit the risk of slip progression through a non-calcified physis. While this is controversial some current relative indications for screw removal include infection, future plans for revision surgery or arthroplasty, screw joint encroachment or symptomatic hardware ${ }^{1}$.

Another controversial aspect to the situ fixation of a SCFE is the decision for prophylactic fixation of the contralateral hip. The rationale for prophylactic pinning comes from the incidence of contralateral SCFE development following being reported as high as $40-80 \%{ }^{4}$. Despite these reported rates other authors advocate long term surveillance until closure of the proximal femoral physis. ${ }^{2,25,26}$. Currently, there are several indications in which authors advocate for prophylactic fixation. The first indication is skeletal immaturity. The major measurement tool for skeletal immaturity and risk of contralateral SCFE formation is the Oxford score which is determined by radiographic appearance of the ilium, greater and lesser trochanter, femoral head and triradiate cartilage. An Oxford score of less than 16 has been shown to $85 \%$ incidence of developing a contralateral SCFE ${ }^{27}$. In addition, presence of an open triradiate cartilage alone has been shown to have an $89 \%$ risk of developing a contralateral SCFE ${ }^{28}$. In addition to skeletal immaturity, patients less than 10 years old are recommended to have prophylactic fixation due to the high incidence of contralateral SCFE ${ }^{28}$. The second major indication for prophylactic fixation is group of patients with associated endocrinopathies. This due to the high prevalence of contralateral involvement with renal insufficiency patients, hypothyroidism and hypogonadism ${ }^{29}$. Overall, in the absence of risk factors, the patient and their guardians should be counselled on the risk of contralateral hip involvement and the need for routine radiographic surveillance following operative management.

In terms of the management of the unstable SCFE, the most commonly treatment is still percutaneous screw fixation and has been shown to have good results ${ }^{30}$. Again the same principles of fixation of a stable SCFE in terms of screw number, size, and positioning. One controversial area of unstable SCFE treatment is whether to perform open reduction with subsequent fixation. Several authors have shown that open reduction utilizing a hip dislocation for exposure reveal significant correction of deformity with low rates of AVN at approximately 1-3 years of follow up ${ }^{31,32}$. These authors concluded that restoration of anatomy aided in the prevention of femoroacetabular impingement and early onset degenerative joint disease ${ }^{31,32}$. The timing of open reduction is appears to be controversial as well with documentation of reduced AVN rates in both immediate (within 24 hours of symptom onset) and delayed ( $<48$ hours) open reduction ${ }^{33,34}$.

The final component of treatment for SCFE is centered on correcting persistent deformities of the proximal femur following fixation and physeal closure. These treatments are considered to improve hip range-of-motion and decrease the incidence of femoroacetabular impingement and subsequent degenerative joint disease. The procedures include proximal femoral osteotomies to correct deformity; either through osteotomies of the proximal or distal femoral neck or intertrochanteric region. Overall, outcomes are varied with studies identify improvement outcomes and good outcomes in as low as $36-40 \%$ of patients $1,35,36$.

In the presence of severe degenerative joint disease following treated or untreated SCFE as a result of AVN or chondrolysis, total hip arthroplasty (THA) can be advocated. Many studies have documented improved function, low complications, and satisfactory implant survival for SCFE patients who undergo THA $42,43,44$.

\section{Complications}

The first major complication that should be monitored for is avascular necrosis (AVN) of the femoral head. The pathophysiology of AVN may be due to disruption of the ascending epiphyseal vessels from the acute tearing of the periosteum as the epiphysis translates posteriorly and inferiorly. The overall rate of AVN has been report 
to be upwards of $4-47 \%{ }^{37}$. However, the risk of AVN appears to be related to multiple risk factors. The first risk factor for AVN is the presence of an unstable SCFE. One study of 240 patients treated with cannulated screw fixation showed a 0\% incidence of AVN in 204 stable SCFEs. Only unstable SCFEs, high grade lesions and patients treated with multiple pins were found to be at risk ${ }^{38}$. Another classic risk factor that is currently controversial is the use of reduction techniques. Finally posterosuperior screw placement may also place the ascending retinacular vessels to the femoral head at risk ${ }^{1}$.

Typically, the patient with AVN will present with progressively worsening pain, deformity and loss of range of motion. Radiographic imaging will typically show early signs of AVN with 12-18 months after initial presentation

39. Early radiographic signs include femoral epiphyseal sclerosis with the traditional sign of subchondral collapse and degenerative joint disease occurring later. Treatment options for AVN include core decompression and other revascularization surgeries in the pre-collapse stage. Post collapse AVN can be approached with reconstructive proximal femoral osteotomies vs hip arthroplasties ${ }^{1}$.

A second postoperative complication is Chondrolysis. Typical incidence of chondrolysis following SCFE fixation is approximately $1-2 \%$ with a predilection in females over males ${ }^{40}$. Current theories on etiology include orthopedic hardware joint penetration, femoroacetabular impingement or autoimmune reaction ${ }^{1}$. The patient will typically present with persistent hip pain and the limb held in flexion, abduction and external rotation. Radiographic imaging will reveal a loss of greater than $50 \%$ of joint space or less than $3 \mathrm{~mm}$ total with associated osteopenia. These radiographic changes typically occur within 1 year of surgery ${ }^{41}$. Bone scans of the hip will reveal inflammation on both the femoral and acetabular sides of the joint. Treatment of this condition is usually symptomatic management including supportive treatment with NSAIDs and physical therapy after the surgeon has ruled out entities such as infection of hardware complication. Overall, patients typically become pain free but persistent hip stiffness has been documented ${ }^{41}$.

\section{DisCUSSION/CONCLUSION}

Slipped capital femoral epiphysis (SCFE) is condition seen in the adolescent population with rising incidence related to the obesity epidemic. Early diagnosis and proper treatment of SCFE is crucial for the long-term health of the hip joint. It is also important to understand the risk factors for contralateral slips and either closely monitor or treat these patients prophylactically. For patients with severe slips or those that suffer from AVN, impingement, or chondrolysis hip preservation interventions can be implemented. For patients that go on to have progressive hip degeneration at a young age, total hip arthroplasty has been shown to be a safe and effective treatment.

\section{REFERENCES}

1. "Slipped Capital Femoral Epiphysis." Tachdjian's Pediatric Orthopaedics from the Texas Scottish Rite Hospital for Children, by John Herring, 2014, pp. 630-665.

2. Loder RT: The demographics of slipped capital femoral epiphysis. An international multicenter study. Clin Orthop Relat Res 1996; 322: pp. 8.

3. Exner GU: Growth and pubertal development in slipped capital femoral epiphysis: a longitudinal study. J Pediatr Orthop 1986; 6: pp. 403

4. Hagglund G, Hansson LI, and Ordeberg G: Epidemiology of slipped capital femoral epiphysis in southern Sweden. Clin Orthop Relat Res 1984; 191: pp. 82

5. "General Principles of Managing Orthopaedic Injuries." Tachdjian's Pediatric Orthopaedics from the Texas Scottish Rite Hospital for Children, by John Herring, 2014, pp. 1199-1223. 
6. Ebraheim, Nabil. "Growth Plate Injuries." Life, Huffington Post, 3 Feb. 2017, www.huffpost.com/entry/ growth-plate-injuries_b_14598372.

7. Bertrand, Todd, et al. "Vascularized Fibular Grafts for Avascular Necrosis After Slipped Capital Femoral Epiphysis: Is Hip Preservation Possible?" Clinical Orthopaedics and Related Research@, vol. 471, no. 7, 2013, pp. 2206-2211.

8. Halverson, Schuyler J., et al. "Leptin Elevation as a Risk Factor for Slipped Capital Femoral Epiphysis Independent of Obesity Status." The Journal of Bone and Joint Surgery, vol. 99, no. 10, 2017, pp. 865-872.

9. Peck, David. "Slipped Capital Femoral Epiphysis: Diagnosis and Management." AAFP Home, 1 Aug. 2010, www.aafp.org/afp/2010/0801/p258.html.

10. Southwick WO: Osteotomy through the lesser trochanter for slipped capital femoral epiphysis. J Bone Joint Surg Am 1967; 49: pp. 807

11. Jarrett, D. Y., Matheney, T., \& Kleinman, P. K. (2013). Imaging SCFE: diagnosis, treatment and complications. Pediatric radiology, 43(1), 71-82.

12. Cohen, M. S., Gelberman, R. H., Griffin, P. P., Kasser, J. R., Emans, J. B., \& Millis, M. B. (1986). Slipped capital femoral epiphysis: assessment of epiphyseal displacement and angulation. Journal of pediatric orthopedics, 6(3), 259-264.

13. Rhoad, R. C., Davidson, R. S., Heyman, S., Dormans, J. P., \& Drummond, D. S. (1999). Pretreatment bone scan in SCFE: a predictor of ischemia and avascular necrosis. Journal of Pediatric Orthopaedics, 19(2), 164-168.

14. Umans, H., Liebling, M. S., Moy, L., Haramati, N., Macy, N. J., \& Pritzker, H. A. (1998). Slipped capital femoral epiphysis: a physeal lesion diagnosed by MRI, with radiographic and CT correlation. Skeletal radiology, 27(3), 139-144.

15. Birch, J. G. (1987). Slipped capital femoral epiphysis: still an emergency. Journal of pediatric orthopedics, 7(3), 334-337.

16. Rattey, T., Piehl, F., \& Wright, J. G. (1996). Acute slipped capital femoral epiphysis. Review of outcomes and rates of avascular necrosis. JBJS, 78(3), 398-402.

17. Lindaman, L. M., Canale, T. S., Beaty, J. H., \& Warner, W. C. (1991). A fluoroscopic technique for determining the incision site for percutaneous fixation of slipped capital femoral epiphysis. Journal of Pediatric Orthopaedics, 11(3), 397-401.

18. Aronson, D. D., \& Carlson, W. E. (1992). Slipped capital femoral epiphysis. A prospective study of fixation with a single screw. JBJS, 74(6), 810-819.

19. Koval, K. J., Lehman, W. B., Rose, D., Koval, R. P., Grant, A., \& Strongwater, A. (1989). Treatment of slipped capital femoral epiphysis with a cannulated-screw technique. The Journal of bone and joint surgery. American volume, 71(9), 1370-1377.

20. Stevens, D. B., Short, B. A., \& Burch, J. M. (1996). In situ fixation of the slipped capital femoral epiphysis with a single screw. Journal of pediatric orthopedics. Part B, 5(2), 85-89.

21. Rostoucher, P., Bensahel, H., Pennecot, G. F., Kaewpornsawan, K., \& Mazda, K. (1996). Slipped capital femoral epiphysis: evaluation of different modes of treatment. JOURNAL OF PEDIATRIC ORTHOPAEDICS PART B, 5 , 96-101.

22. Stambough, J. L., Davidson, R. S., Ellis, R. D., \& Gregg, J. R. (1986). Slipped capital femoral epiphysis: an analysis of 80 patients as to pin placement and number. Journal of pediatric orthopedics, 6(3), 265-273. 
23. Karol, L. A., Doane, R. M., Cornicelli, S. F., Zak, P. A., \& Haut, R. C. (1992). Single versus double screw fixation for treatment of slipped capital femoral epiphysis: a biomechanical analysis. Journal of pediatric orthopedics, 12(6), 741-745.

24. Carney, B. T., Birnbaum, P., \& Minter, C. (2003). Slip progression after in situ single screw fixation for stable slipped capital femoral epiphysis. Journal of Pediatric Orthopaedics, 23(5), 584-589.

25. Jerre, R., Billing, L., Hansson, G., \& Wallin, J. (1994). The contralateral hip in patients primarily treated for unilateral slipped upper femoral epiphysis. Long-term follow-up of 61 hips. Bone \& Joint Journal, 76(4), 563-567.

26. Loder, R. T., Aronson, D. D., \& Greenfield, M. L. (1993). The epidemiology of bilateral slipped capital femoral epiphysis. A study of children in Michigan. JBJS, 75(8), 1141-1147.

27. Popejoy, D., Emara, K., \& Birch, J. (2012). Prediction of contralateral slipped capital femoral epiphysis using the modified Oxford bone age score. Journal of Pediatric Orthopaedics, 32(3), 290-294

28. Stasikelis, P. J., Sullivan, C. M., Phillips, W. A., \& Polard, A. J. (1996). Slipped capital femoral epiphysis: prediction of contralateral involvement. JBJS, 78(8), 1149-1155.

29. Loder, R. T., Wittenberg, B., \& DeSilva, G. (1995). Slipped capital femoral epiphysis associated with endocrine disorders. Journal of pediatric orthopedics, 15(3), 349-356.

30. Goodman, W. W., Johnson, J. T., \& Robertson Jr, W. W. (1996). Single screw fixation for acute and acute-onchronic slipped capital femoral epiphysis. Clinical orthopaedics and related research, (322), 86-90

31. Slongo, T., Kakaty, D., Krause, F., \& Ziebarth, K. (2010). Treatment of slipped capital femoral epiphysis with a modified Dunn procedure. JBJS, 92(18), 2898-2908.

32. Ziebarth, K., Zilkens, C., Spencer, S., Leunig, M., Ganz, R., \& Kim, Y. J. (2009). Capital realignment for moderate and severe SCFE using a modified Dunn procedure. Clinical orthopaedics and related research, 467(3), 704-716.

33. Loder, R. T., Richards, S. B., Shapiro, P. S., Reznick, L. R., \& Aronson, D. D. (1993). the Importance of Physeal Stability. JBJS, 75(8), 1134-1140.

34. Peterson, M. D., Weiner, D. S., Green, N. E., \& Terry, C. L. (1997). Acute slipped capital femoral epiphysis: the value and safety of urgent manipulative reduction. Journal of Pediatric Orthopaedics, 17(5), 648-654.

35. Jerre, R., Hansson, G. O. R. A. N., Wallin, J., \& Karlsson, J. (1996). Long-term results after realignment operations for slipped upper femoral epiphysis. J Bone Joint Surg Br, 78(5), 745-750.

36. Carney, B. T., Weinstein, S. L., \& Noble, J. (1991). Long-term follow-up of slipped capital femoral epiphysis. The Journal of bone and joint surgery. American volume, 73(5), 667-674.

37. Loder, R. T. (2001). Unstable slipped capital femoral epiphysis. Journal of Pediatric Orthopaedics, 21(5), 694-699.

38. Tokmakova, K. P., Stanton, R. P., \& Mason, D. E. (2003). Factors influencing the development of osteonecrosis in patients treated for slipped capital femoral epiphysis. JBJS, 85(5), 798-801.

39. Krahn, T. H., Canale, S. T., Beaty, J. H., Warner, W. C., \& Lourenco, P. (1993). Long-term follow-up of patients with avascular necrosis after treatment of slipped capital femoral epiphysis. Journal of pediatric orthopedics, 13(2), 154-158.

40. Kennedy, J. P., \& Weiner, D. S. (1990). Results of slipped capital femoral epiphysis in the black population. Journal of pediatric orthopedics, 10(2), 224-227. 
41. Vrettos, B. C., \& Hoffman, E. B. (1993). Chondrolysis in slipped upper femoral epiphysis. Long-term study of the aetiology and natural history. Bone \& Joint Journal, 75(6), 956-961.

42. Traina, Francesco, et al. "Outcomes of Total Hip Replacement in Patients with Slipped Capital Femoral Epiphysis." Archives of Orthopaedic and Trauma Surgery, vol. 132, no. 8, 2012, pp. 1133-1139.

43. Lehmann, Trude G, et al. "Total Hip Arthroplasty in Young Adults, with Focus on Perthes' Disease and Slipped Capital Femoral Epiphysis." Acta Orthopaedica, vol. 83, no. 2, 2011, pp. 159-164.

44. Larson, Annalise Noelle, et al. "Avascular Necrosis Most Common Indication for Hip Arthroplasty in Patients With Slipped Capital Femoral Epiphysis." Journal of Pediatric Orthopaedics, vol. 30, no. 8, 2010, pp. 767-773.

Citation: Rachel Michael, MD, Christopher Jackson, MD, Briana Stirling, MD, Vithal Shendge, MD. "Slipped Capital Femoral Epiphysis: A Case Report and Comprehensive Review of Current Literature". American Research Journal of Orthopedics and Traumatology. 2019; 4(1): 1-16.

Copyright (c) 2019 Rachel Michael, MD, Christopher Jackson, MD, Briana Stirling, MD, Vithal Shendge, MD. This is an open access article distributed under the Creative Commons Attribution License, which permits unrestricted use, distribution, and reproduction in any medium, provided the original work is properly cited. 